\title{
SPECTRAL ANALYSIS FOR THE GENERALIZED HERMITE POLYNOMIALS
}

\author{
ALLAN M. KRALL
}

\begin{abstract}
The operator theory associated with the Hermite polynomials does not extend to the generalized Hermite polynomials because the even and odd polynomials satisfy different differential equations. We show that this leads to two problems, each of interest on its own. We then weld them together to form a united spectral expansion. In addition, the exponent $\mu$ in the weight $|x|^{2 \mu} e^{-x^{2}}$ has traditionally always been greater than $-\frac{1}{2}$. We show what happens if $\mu \leq-\frac{1}{2}$. Finally, we examine the differential equations in left-definite spaces.
\end{abstract}

\section{INTRODUCTION}

The generalized Hermite polynomials $\left\{H_{n}^{(\mu)}\right\}_{n=0}^{\infty}$, given by

$$
H_{2 n}^{(\mu)}(x)=\sum_{k=0}^{n}\left(\begin{array}{c}
n+\mu-\frac{1}{2} \\
n-k
\end{array}\right)(-1)^{n-k} 2^{2 n} n ! x^{2 k} / k !
$$

and

$$
H_{2 n+1}^{(\mu)}(x)=\sum_{k=0}^{n}\left(\begin{array}{c}
n+\mu+\frac{1}{2} \\
n-k
\end{array}\right)(-1)^{n-k} 2^{2 n+1} n ! x^{2 k+1} / k !,
$$

satisfy two differential equations

$$
x y^{\prime \prime}+2\left(\mu-x^{2}\right) y^{\prime}+[2(2 n) x] y=0,
$$

and

$$
x y^{\prime \prime}+2\left(\mu-x^{2}\right) y^{\prime}+\left[2(2 n+1) x-2 \mu x^{-1}\right] y,
$$

resp. [1]. While they form a complete orthogonal set in $L^{2}\left(-\infty, \infty ;|x|^{2 \mu} e^{-x^{2}}\right)$, $\mu>-\frac{1}{2}$. The presence of two differential equations precludes the existence of an operator theoretic foundation such as found for the ordinary Hermite polynomials, $\left\{H_{n}^{(0)}\right\}_{n=0}^{\infty}$, found when $\mu=0$. This should not be looked on as a failure, however, but as a twofold opportunity. Indeed, both equations are singular, not only at $\infty$, but also at 0 , and so an operator theory may be developed separately for each on $(0, \infty)$, on which the even and odd degree polynomials each separately form a compete orthogonal set.

It is this theory we wish to develop here not only for $\mu>-\frac{1}{2}$, but also for $\mu \leq-\frac{1}{2}$. Then we wish to further extend it to what are called left-definite

Received by the editors November 2, 1992.

1991 Mathematics Subject Classification. Primary 33C45; Secondary 34B20. 
spaces. These are generated by the differential equations as well, also involve the first derivative, and are, therefore, Sobolev spaces. Because very much to follow is closely related to known results, we will frequently merely give references for proofs. We hope, however, to present a clear picture of what occurs.

We remark that the polynomials $\left\{H_{n}^{(\mu)}(x)\right\}_{n=0}^{\infty}, \mu<-\frac{1}{2}$, have been examined in a Krein space [5], where they also form a complete orthogonal set.

\section{2. $L^{2}$ THEORY FOR THE POLYNOMIALS OF EVEN DEGREE}

The classic orthogonality condition found in [1] becomes

$$
\int_{0}^{\infty} H_{2 n}^{(\mu)}(x) H_{2 m}^{(\mu)}(x)|x|^{2 \mu} e^{-x^{2}} d x=2^{4 n-1} n ! \Gamma(n+\mu+1) \delta_{m n},
$$

when the polynomials are of even degree, $\mu>-\frac{1}{2}$. The differential equation in formally selfadjoint form

$$
l y=\frac{1}{2}\left[-\left(x^{2 \mu} e^{-x^{2}}\right)^{-1}\left(x^{2 \mu} e^{-x^{2}} y^{\prime}\right)^{\prime}\right]=(2 n) y
$$

can be put in Liouville normal form by setting

$$
y=x^{-\mu} e^{x^{2} / 2} u
$$

The result is that $l$ becomes isomorphic to

$$
\tilde{l} u=-\frac{1}{2}\left(u^{\prime \prime}-\left[\mu(\mu-1) x^{-2}-(2 \mu-1)+x^{2}\right] u\right)=(2 n) u
$$

on $L^{2}(0, \infty ; 1)$. Examination of this expression quickly establishes that $\infty$ is in the limit point case [2, p. 229]. That is, no boundary condition at $\infty$ is required.

Further, the substitution $t=x^{2}$ transforms the original differential equation into

$$
t \frac{d^{2} y}{d t^{2}}+\left(\frac{1}{2}+\mu-t\right) \frac{d y}{d t}+n y=0
$$

Letting $\alpha=\mu-\frac{1}{2}$, this is

$$
t \frac{d^{2} y}{d t^{2}}+(1+\alpha-t) \frac{d y}{d t}+n y=0
$$

the generalized Laguerre equation. As is well known [1, 11],

$$
y=H_{2 n}^{(\mu)}(x)=C_{n} L_{n}^{\left(\mu-\frac{1}{2}\right)}(t)
$$

where $C_{n}=(-1)^{n} 2^{2 n} n !$.

Evaluation of the boundary $t=0$ is well known for the Laguerre polynomials [4]. The polynomial generating boundary condition is

$$
\lim _{t \rightarrow 0} t^{\alpha+1} e^{-t} \frac{d y}{d t}=0
$$

This is equivalent to

$$
\lim _{x \rightarrow 0} x^{2 \mu} e^{-x^{2}} \frac{d y}{d x}=-\lim _{x \rightarrow 0} x^{2 \mu} e^{-x^{2}} W[y, 1]=0
$$


in the Hermite case. Clearly the even Hermite polynomials satisfy this boundary condition at $x=0$.

The Laguerre boundary condition is needed (limit-circle case [2]) when $-1<$ $\alpha<1$. This is equivalent to $-\frac{1}{2}<\mu<\frac{3}{2}$. This is consistent with direct findings, using the method of Frobenius: Indicial roots at $x=0$ are $r=0$ and $r=1-2 \mu$. The first indicial root, 0 , generates a solution which is in $L^{2}\left(0,1 ; x^{2 \mu} c^{-x^{2}}\right)$ when $\mu>-\frac{1}{2}$. The second root, $1-2 \mu$, generates a solution which is in $L^{2}\left(0,1 ; x^{2 \mu} e^{-x^{2}}\right)$ when $\mu<\frac{3}{2}$.

With this preliminary information, the even boundary value problem and operator theory is easily described.

2.1. Definition. We denote by $D_{e}$ those elements $y$ in $L^{2}\left(0, \infty ; x^{2 \mu} e^{-x^{2}}\right)$ which satisfy the following.

(a) $y$ is absolutely continuous on all closed subintervals of $(0, \infty)$.

(b) $x^{2 \mu} e^{-x^{2}} y^{\prime}$ is absolutely continuous on all closed subintervals of $(0, \infty)$.

(c) $l y=-\frac{1}{2}\left(x^{2 \mu} e^{-x^{2}}\right)^{-1}\left(x^{2 \mu} e^{-x^{2}} y^{\prime}\right)^{\prime}$ exists almost everywhere and is in $L^{2}\left(0, \infty ; x^{2 \mu} e^{-x^{2}}\right)$.

(d) $\lim _{x \rightarrow 0} x^{2 \mu} e^{-x^{2}} y^{\prime}(x)=0$.

We define the operator $L$ by setting $L y=l y$ for all $y$ in $D_{e}$.

2.2. Theorem. $L$ is selfadjoint in $L^{2}\left(0, \infty ; x^{2 \mu} e^{-x^{2}}\right)$.

This is well known. The verification of this and many other related facts may be found in $[2,10]$

2.3. Theorem. The spectrum of $L$ is discrete, consisting of the even integers $\{2 n\}_{n=0}^{\infty}$. Associated with the eigenvalue $2 n$ is the eigenfunction $H_{2 n}^{(\mu)}(x)$, $n=0,1, \cdots$. These eigenfunctions form a complete orthogonal set in $L^{2}\left(0, \infty ; x^{2 \mu} e^{-x^{2}}\right)$.

Proof. It is sufficient to show that the even generalized Hermite polynomials form a complete orthogonal set. Suppose there is an element $z$ orthogonal to them all. Then

$$
\int_{0}^{\infty} \bar{z}(x) H_{2 n}^{(\mu)}(x) x^{2 \mu} e^{-x^{2}} d x=0, \quad n=0,1, \cdots
$$

We extend $z$ to $(-\infty, 0)$ as an even function. Then

$$
\int_{\infty}^{-\infty} \bar{z}(x) H_{n}^{(\mu)}(x)|x|^{2 \mu} e^{-x^{2}} d x=0, \quad n=0,1, \cdots
$$

By rearrangement of terms, this implies

$$
\int_{\infty}^{-\infty}\left[\bar{z}(x)|x|^{2 \mu}\right] H_{k}^{(0)}(x) e^{-x^{2}} d x=0, \quad n=0,1, \cdots
$$

and so $z(x)|x|^{2 \mu}=0$ in $L^{2}\left(-\infty, \infty ; e^{-x^{2}}\right)$. This implies $z=0$ a.e. and therefore also in $L^{2}\left(0, \infty ; x^{2 \mu} e^{-x^{2}}\right)$. 
2.4. Corollary. Let $f$ be an element in $L^{2}\left(0, \infty ; x^{2 \mu} e^{-x^{2}}\right)$. Let $y$ be in $D_{e}$. Then

$$
\begin{gathered}
f(x)=\sum_{n=0}^{\infty} c_{2 n} H_{2 n}^{(\mu)}(x), \\
(L-\lambda)^{-1} f(x)=\sum_{n=0}^{\infty} \frac{c_{2 n}}{2 n-\lambda} H_{2 n}^{(\mu)}(x), \quad \lambda \neq 2 n,
\end{gathered}
$$

where

$$
c_{2 n}=\frac{\int_{0}^{\infty} f(\xi) H_{2 n}^{(\mu)}(\xi) \xi^{2 \mu} e^{-\xi^{2}} d \xi}{2^{4 n-1} n ! \Gamma(n+\mu+1)}
$$

and

$$
L y(x)=\sum_{n=0}^{\infty}(2 n) d_{2 n} H_{2 n}^{(\mu)}(x),
$$

where

$$
d_{2 n}=\frac{\int_{0}^{\infty} y(\xi) H_{2 n}^{(\mu)}(\xi) \xi^{2 \mu} e^{-\xi^{2}} d \xi}{2^{4 n-1} n ! \Gamma(n+\mu+1)}
$$

\section{3. $L^{2}$ THEORY FOR THE POLYNOMIALS OF ODD DEGREE}

The classic orthogonality condition found in [1] becomes

$$
\int_{0}^{\infty} H_{2 n+1}^{(\mu)}(x) H_{2 m+1}^{(\mu)}(x)|x|^{2 \mu} e^{-x^{2}} d x=2^{4 n+1} n ! \Gamma\left(n+\mu+\frac{3}{2}\right) \delta_{m n},
$$

when the polynomials are of odd degree, $\mu>-3 / 2$. The differential equation, in formally selfadjoint form,

$$
m y=\frac{1}{2}\left[\left(x^{2 \mu} e^{-x^{2}}\right)^{-1}\left(-\left(x^{2 \mu} e^{-x^{2}} y^{\prime}\right)^{\prime}+2 \mu x^{2 \mu-2} e^{-x^{2}} y\right)\right]=(2 n+1) y
$$

can be put in Liouville normal form by setting

$$
y=x^{-\mu} e^{x^{2} / 2} u \text {. }
$$

The result is that $m$ becomes isomorphic to

$$
\tilde{m} u=-\frac{1}{2}\left(u^{\prime \prime}-\left[\mu(\mu+1) x^{-2}-(2 \mu+1)+x^{2}\right] u\right)=(2 n+1) u
$$

on $L^{2}(0, \infty ; 1)$. Examination of this expression quickly establishes that $\infty$ is again in the limit point case [2, p. 229]. That is, no boundary condition at $\infty$ is required.

Further the substitutions $t=x^{2}, y=\sqrt{t} z$ transform the original differential equation into

$$
t \frac{d^{2} z}{d t^{2}}+\left(\mu+\frac{3}{2}-t\right) \frac{d z}{d t}+n z=0
$$

Letting $\alpha=\mu+\frac{1}{2}$, this is

$$
t \frac{d^{2} z}{d t^{2}}+(1+\alpha-t) \frac{d z}{d t}+n z=0
$$

the generalized Laguerre equation. As is well known $[1,11]$ 


$$
y=H_{2 n+1}^{\mu}(x)=C_{n} \sqrt{t} L_{n}^{\left(\mu+\frac{1}{2}\right)}(t),
$$

where $C_{n}=(-1)^{n} 2^{2 n+1} n !$.

Evaluation of the boundary $t=0$ is well known for the Laguerre polynomials [4]. The polynomial generating boundary condition is

$$
\lim _{t \rightarrow 0} t^{\alpha+1} e^{-t} \frac{d z}{d t}=0
$$

This is equivalent to

$$
\lim _{x \rightarrow 0} x^{2 \mu} e^{-x^{2}}\left(x \frac{d y}{d x}-y\right)=-\lim _{x \rightarrow 0} x^{2 \mu} e^{-x^{2}} W[y, x]=0
$$

in the Hermite case. Clearly the odd Hermite polynomials satisfy this boundary condition at $x=0$.

The Laguerre boundary conditions needed (limit-circle case [2]) when $-1<$ $\alpha<1$. This is equivalent to $-3 / 2<\mu<1 / 2$. This is consistent with the method of Frobenius: Indicial roots at $x=0$ are $r=1$ and $r=-2 \mu$. The first indicial root, 1 , generates a solution which is in $L^{2}\left(0,1 ; x^{2 \mu} e^{-x^{2}}\right)$ when $\mu>$ $-3 / 2$. The second, $-2 \mu$, generates a solution which is in $L^{2}\left(0,1 ; x^{2 \mu} e^{-x^{2}}\right)$ when $\mu<1 / 2$.

With this preliminary information, the odd boundary value problem and operator theory is easily described.

3.1. Definition. We denote by $D_{0}$ those elements $y$ in $L^{2}\left(0, \infty ; x^{2 \mu} e^{-x^{2}}\right)$ which satisfy the following.

(a) $y$ is absolutely continuous on all closed subintervals of $(0, \infty)$.

(b) $x^{2 \mu} e^{-x^{2}} y^{\prime}$ is absolutely continuous on all closed subintervals of $(0, \infty)$.

(c) $m y=-\frac{1}{2}\left(x^{2 \mu} e^{-x^{2}}\right)^{-1}\left(\left(x^{2 \mu} y^{\prime}\right)^{\prime}-2 \mu x^{2 \mu-2} e^{-x^{2}} y\right)$ exists a.e. and is in $L^{2}\left(0, \infty ; x^{2 \mu} e^{-x^{2}}\right)$.

(d) $\lim _{x \rightarrow 0} x^{2 \mu} e^{-x^{2}}\left(x y^{\prime}(x)-y(x)\right)=0$.

We define the operator $M$ by setting $M y=m y$ for all $y$ in $D_{0}$.

3.2. Theorem. $M$ is selfadjoint in $L^{2}\left(0, \infty ; x^{2 \mu} e^{-x^{2}}\right)$.

Proof. Again see [2, 10].

3.3. Theorem. The spectrum of $M$ is discrete, consisting of the odd integers $\{2 n+1\}_{n=0}^{\infty}$. Associated with the eigenvalue $2 n+1$ is the eigenfunction $H_{2 n+1}^{(\mu)}(x)$, $n=0,1, \cdots$. These eigenfunctions form a complete orthogonal set in $L^{2}\left(0, \infty ; x^{2 \mu} e^{-x^{2}}\right)$.

Proof. Comparing the proof of Theorem 2.3, the only change is that $z$ should be extended as an odd function.

3.4. Corollary. Let $f$ be an element in $L^{2}\left(0, \infty ; x^{2 \mu} e^{-x^{2}}\right)$. Let $y$ be in $D_{0}$. Then

$$
\begin{gathered}
f(x)=\sum_{n=0}^{\infty} c_{2 n+1} H_{2 n+1}^{(\mu)}(x), \\
(M-\lambda)^{-1} f=\sum_{n=0}^{\infty} \frac{c_{2 n+1}}{2 n+1-\lambda} H_{2 n+1}^{(\mu)}(x), \quad \lambda \neq 2 n+1,
\end{gathered}
$$


where

$$
c_{2 n+1}=\frac{\int_{\infty}^{-\infty} f(\xi) H_{2 n+1}^{(\mu)}(\xi) \xi^{2 \mu} e^{-\xi^{2}} d \xi}{2^{4 n+1} n ! \Gamma\left(n+\mu+\frac{3}{2}\right)}
$$

and

$$
M y(x)=\sum_{n=0}^{\infty}(2 n+1) d_{2 n+1} H_{2 n+1}^{(\mu)}(x)
$$

where

$$
d_{2 n+1}=\frac{\int_{\infty}^{-\infty} y(\xi) H_{2 n+1}^{(\mu)}(\xi) \xi^{2 \mu} e^{-\xi^{2}} d \xi}{2^{4 n+1} n ! \Gamma\left(n+\mu+\frac{3}{2}\right)}
$$

\section{4. $\quad L^{2}$ THEORY ON $(-\infty, \infty)$}

We consider the Hilbert space $L^{2}\left(-\infty, \infty ;|x|^{2 \mu} e^{\left.-x^{2} / 2\right)}, \mu>-1 / 2\right.$. Each $f$ in that space corresponds to two orthogonal functions

$$
f_{e}(x)=\frac{1}{2}(f(x)+f(-x)), \quad f_{0}(x)=\frac{1}{2}(f(x)-f(-x)) .
$$

Collapsing the norms of $f_{e}$ and $f_{0}$ onto $(0, \infty)$, we have

$$
\int_{0}^{\infty}|f(x)|^{2} \frac{|x|^{2 \mu} e^{-x^{2}}}{2} d x=\int_{0}^{\infty}\left|f_{e}(x)\right|^{2} x^{2 \mu} e^{-x^{2}} d x+\int_{0}^{\infty}\left|f_{0}(x)\right|^{2} x^{2 \mu} e^{-x^{2}} d x .
$$

Thus the Hilbert space on $(-\infty, \infty)$ is isomorphic to the tensor product of $L^{2}\left(0, \infty ; x^{2 \mu} e^{-x^{2}}\right)$ with itself. We identify with $f$ on $(-\infty, \infty)$ the vector $\left(\begin{array}{l}f_{e} \\ f_{0}\end{array}\right)$, each component defined on $(0, \infty)$.

We can define a matrix differential operator $P$ by setting

$$
P y \simeq\left(\begin{array}{cc}
L & 0 \\
0 & M
\end{array}\right)\left(\begin{array}{l}
y_{e} \\
y_{0}
\end{array}\right)=\left(\begin{array}{c}
L y_{e} \\
M y_{0}
\end{array}\right) .
$$

The domain of $P$ is clearly those $y$ for which $y_{e}$ is in $D_{e}, y_{0}$ is in $D_{0}$. The spectrum of $P$ is discrete, consisting of the nonnegative integers. Eigenvectors for $P$ fall into two classes; $\left\{\left(\begin{array}{c}H_{2 n}^{(\mu)} \\ 0\end{array}\right)\right\}_{n=0}^{\infty}$, with eigenvalues $\{2 n\}_{n=1}^{\infty}$, and $\left\{\left(\begin{array}{c}0 \\ H_{2 n+1}^{(\mu)}\end{array}\right)\right\}_{n=0}^{\infty}$, with eigenvalues $\{2 n+1\}_{n=0}^{\infty}$. We denote the first set by $\left\{E_{2 n}\right\}_{n=0}^{\infty}$, the second by $\left\{E_{2 n+1}\right\}_{n=0}^{\infty}$.

For each $f$ in $L^{2}\left(-\infty, \infty ;|x|^{2 \mu} e^{-x^{2}} / 2\right)$, we have

$$
\left(\begin{array}{l}
f_{e} \\
f_{0}
\end{array}\right)=\sum_{n=0}^{\infty} A_{n} E_{n}(x)
$$

where

$$
A_{2 n}=\frac{\int_{0}^{\infty} f_{e}(\xi) H_{2 n}^{(\mu)}(\xi) \xi^{2 \mu} e^{-\xi^{2}} d \xi}{2^{4 n-1} n ! \Gamma(n+\mu+1)}
$$

and

$$
A_{2 n+1}=\frac{\int_{0}^{\infty} f_{0}(\xi) H_{2 n+1}^{(\mu)}(\xi) \xi^{2 \mu} e^{-\xi^{2}} d \xi}{2^{4 n+1} n ! \Gamma\left(n+\mu+\frac{3}{2}\right)}
$$


If $y$ is in the domain of $P$, then

$$
P y=\left(\begin{array}{c}
L y_{e} \\
M y_{0}
\end{array}\right)=\sum_{n=0}^{\infty} n A_{n} E_{n}(x) .
$$

For each $f$ in $L^{2}\left(-\infty, \infty ;|x|^{2 \mu} e^{-x^{2}} / 2\right)$

$$
(P-\lambda)^{-1} f=\sum_{n=0}^{\infty} \frac{A_{n}}{n-\lambda} E_{n}(x) .
$$

If $f_{e}$ and $f_{0}$ are added together, then

$$
f(x)=\sum_{n=0}^{\infty} A_{n} H_{n}^{(\mu)}(x) .
$$

This addition of components, however, precludes the development of an operator theory except when $\mu=0$.

\section{SHIFTED SPACES}

Throughout the previous sections the requirement $\mu>-1 / 2$ or $\mu>-3 / 2$ was made in order to ensure that the Hermite polynomials were in the space $L^{2}\left(0, \infty ; x^{2 \mu} e^{-x}\right)$. There is the age old question of what happens if $\mu \leqq-1 / 2$ or $\mu \leqq-3 / 2$ instead. We show that a simple change in the dependent variable changes the even differential equation into the odd differential equation, but with the negative $\mu$ now replaced by a positive parameter. Likewise the odd differential equation changes into the even differential equation, but with the negative $\mu$ again replaced by a positive parameter.

The even problem. Let us consider the even equation

$$
x y^{\prime \prime}+2\left(\mu-x^{2}\right) y^{\prime}+[2(2 n) x] y=0 .
$$

If $y=x^{-2 \mu} Y$, the result is

$$
x Y^{\prime \prime}+2\left([-\mu]-x^{2}\right) Y^{\prime}+\left[2(2 n+2 \mu) x-2[-\mu] x^{-1}\right] Y=0 .
$$

If $2 n+2 \mu=2 r+1$, and $\mu=-\nu$, the resulting equation

$$
x Y^{\prime \prime}+2\left(\nu-x^{2}\right) Y^{\prime}+\left[2(2 r+1) x-2 \nu x^{-1}\right] Y=0
$$

is the standard odd equation.

The standard odd boundary condition at $x=0$,

$$
\lim _{x \rightarrow 0} x^{2 \nu} e^{-x^{2}}\left(x Y^{\prime}(x)-Y(x)\right)=0,
$$

is equivalent to the condition

$$
\lim _{x \rightarrow 0} e^{-x^{2}}\left(x y^{\prime}(x)+[2 \mu-1] y(x)\right)=0 .
$$

It is easy to see that under the transformation $y=x^{-2 \mu} Y$, the space $L^{2}\left(0, \infty ; x^{2 \mu} e^{-x^{2}}\right)$ is isomorphic to $L^{2}\left(0, \infty ; x^{2 \nu} e^{-x^{2}}\right)$, where $\mu=-\nu$. Furthermore, the operator $l$ on $y$ corresponds to the operator $m-2 \mu$ on $Y$.

Transforming the odd equation results from $Y$ to $y$ gives the following when $\mu<3 / 2$. 
5.1. Definition. We denote by $\widetilde{D}_{e}$ those elements $y$ in $L^{2}\left(0, \infty ; x^{2 \mu} e^{-x^{2}}\right)$ which satisfy the following.

(a) $y$ is absolutely continuous on all closed subsets of $(0, \infty)$.

(b) $x^{2 \mu} e^{-x^{2}} y^{\prime}$ is absolutely continuous on all closed subintervals of $(0, \infty)$.

(c) $l y=-\frac{1}{2}\left(x^{2 \mu} e^{-x^{2}}\right)^{-1}\left(x^{2 \mu} e^{-x^{2}} y^{\prime}\right)^{\prime}$ exists almost everywhere and is in $L^{2}\left(0, \infty ; x^{2 \mu} e^{-x^{2}}\right)$.

(d) $\lim _{x \rightarrow 0} e^{-x^{2}}\left(x y^{\prime}(x)+[2 \mu-1] y(x)\right)=0$

We define the operator $\widetilde{L}$ by setting $\widetilde{L} y=l y$ for all $y$ in $\widetilde{D}_{e}$.

5.2. Theorem. $\widetilde{L}$ is selfadjoint in $L^{2}\left(0, \infty ; x^{2 \mu} e^{-x^{2}}\right)$.

5.3. Theorem. The spectrum of $\widetilde{L}$ is discrete, consisting of the numbers $\{2 n+$ $1-2 \mu\}_{n=0}^{\infty}$. Associated with each eigenvalue $2 n+1-2 \mu$ is the eigenfunction $x^{-2 \mu} H_{2 n+1}^{(-\mu)}(x), n=0,1, \cdots$. These eigenvalues form a complete orthogonal set in $L^{2}\left(0, \infty ; x^{2 \mu} e^{-x^{2}}\right)$.

5.4. Corollary. Let $f$ be any element in $L^{2}\left(0, \infty ; x^{2 \mu} e^{-x^{2}}\right)$. Let $y$ be in $\widetilde{D}_{e}$. Then

$$
\begin{gathered}
f(x)=\sum_{n=0}^{\infty} \widetilde{c}_{2 n} x^{-2 \mu} H_{2 n+1}^{(-\mu)}(x), \\
(\widetilde{L}-\lambda)^{-1} f(x)=\sum_{n=0}^{\infty} \frac{\widetilde{c}_{2 n+1}}{2 n+1-2 \mu-\lambda} x^{-2 \mu} H_{2 n+1}^{(-\mu)}(x), \quad \lambda \neq 2 n+1-2 \mu,
\end{gathered}
$$

where

$$
\tilde{c}_{2 n+1}=\frac{\int_{0}^{\infty} f(\xi)\left(\xi^{-2 \mu} H_{2 n+1}^{(-\mu)}(\xi)\right) \xi^{2 \mu} e^{-\xi^{2}} d \xi}{2^{4 n+1} n ! \Gamma(n-\mu+3 / 2)}
$$

and

$$
\widetilde{L} y(x)=\sum_{n=0}^{\infty}(2 n+1-2 \mu) \widetilde{d}_{2 n+1} x^{-2 \mu} H_{2 n+1}^{(-\mu)}(x),
$$

where

$$
\tilde{d}_{2 n+1}=\frac{\int_{0}^{\infty} y(\xi)\left(\xi^{-2 \mu} H_{2 n+1}^{(-\mu)}(\xi)\right) \xi^{2 \mu} e^{-\xi^{2}} d \xi}{2^{4 n+1} n ! \Gamma(n-\mu+3 / 2)} .
$$

We remark that the transformation $y=x^{1-2 \mu} z$ leads to the same results.

The odd problem. Let us consider the odd equation

$$
x y^{\prime \prime}+2\left(\mu-x^{2}\right) y^{\prime}+\left[2(2 n+1) x-2 \mu x^{-1}\right] y=0 .
$$

If $y=x^{-2 \mu} Y$, the result is

$$
x Y^{\prime \prime}+2\left([-\mu]-x^{2}\right) Y^{\prime}+[2(2 n+1+2 \mu) x] Y=0 .
$$

If $2 n+1+2 \mu=2 r$ and $\mu=-\nu$, the resulting equation

$$
x Y^{\prime \prime}+2\left(\nu-x^{2}\right) Y^{\prime}+[2(2 r) x] Y=0
$$

is the standard even equation. 
The standard even boundary condition

$$
\lim _{x \rightarrow 0} x^{2 \nu} e^{-x^{2}} \frac{d Y}{d x}=0
$$

is equivalent to the condition

$$
\lim _{x \rightarrow 0} e^{-x^{2}}\left(y^{\prime}(x)+2 \mu x^{-1} y(x)\right)=0 .
$$

It is easy to see that under the transformation $y=x^{-2 \mu} Y$, the space $L^{2}\left(0, \infty ; x^{2 \mu} e^{-x^{2}}\right)$ is isomorphic to $L^{2}\left(0, \infty ; x^{2 \nu} e^{-x^{2}}\right)$, where $\mu=-\nu$. Furthermore, the operator $m$ on $y$ corresponds to the operator $l-2 \mu$ on $Y$.

Transforming the even equation results from $Y$ to $y$ gives the following when $\mu<1 / 2$.

5.5. Definition. We denote by $\widetilde{D}_{0}$ those elements $y$ in $L^{2}\left(0, \infty ; x^{2 \mu} e^{-x^{2}}\right)$ which satisfy the following.

(a) $y$ is absolutely continuous on all closed subintervals of $(0, \infty)$.

(b) $x^{2 \mu} e^{-x^{2}} y^{\prime}$ is absolutely continuous on all closed subintervals $(0, \infty)$.

(c) $m y=-\frac{1}{2}\left(x^{2 \mu} e^{-x^{2}}\right)^{-1}\left(\left(x^{2 \mu} e^{-x^{2}} y^{\prime}\right)^{\prime}-2 \mu x^{2 \mu-2} e^{-x^{2}} y\right)$ exists a.e. and is in $L^{2}\left(0, \infty ; x^{2 \mu} e^{-x^{2}}\right)$.

(d) $\lim _{x \rightarrow 0} e^{-x^{2}}\left(y^{\prime}(x)+2 \mu x^{-1} y(x)\right)=0$.

We define the operator $\widetilde{M}$ by setting $\widetilde{M} y=m y$ for all $y$ in $\widetilde{D}_{0}$.

5.6. Theorem. $\widetilde{M}$ is selfadjoint in $L^{2}\left(0, \infty ; x^{2 \mu} e^{-x^{2}}\right)$.

5.7. Theorem. The spectrum of $\widetilde{M}$ is discrete, consisting of $\{2 n-2 \mu\}_{n=0}^{\infty}$. Associated with each eigenvalue $2 n-2 \mu$ is the eigenfunction $x^{-2 \mu} H_{2 n}^{(-\mu)}(x), n=$ $0,1, \cdots$. These eigenvalues form a complete orthogonal set in $L^{2}\left(0, \infty ; x^{2 \mu} e^{-x^{2}}\right)$.

5.8. Corollary. Let $f$ be any element in $L^{2}\left(0, \infty ; x^{2 \mu} e^{-x^{2}}\right)$. Let $y$ be in $\widetilde{D}_{0}$. Then

$$
\begin{gathered}
f(x)=\sum_{n=0}^{\infty} \widetilde{c}_{2 n} x^{-2 \mu} H_{2 n}^{(-\mu)}(x), \\
(\widetilde{M}-\lambda)^{-1} f(x)=\sum_{n=0}^{\infty} \frac{\widetilde{c}_{2 n}}{2 n-2 \mu-\lambda} x^{-2 \mu} H_{2 n}^{(-\mu)}(x), \quad \lambda \neq 2 n-2 \mu,
\end{gathered}
$$

where

$$
\widetilde{c}_{2 n}=\frac{\int_{0}^{\infty} f(\xi)\left(\xi^{-2 \mu} H_{2 n}^{(-\mu)}(\xi)\right) \xi^{2 \mu} e^{-\xi^{2}} d \xi}{2^{4 n-1} n ! \Gamma(n-\mu+1)},
$$

and

$$
\widetilde{M} y(x)=\sum_{n=0}^{\infty}(2 n-2 \mu) \widetilde{\mathscr{D}}_{2 n} x^{-2 \mu} H_{2 n}^{(-\mu)}(x) \text {, }
$$

where

$$
\tilde{d}_{2 n}=\frac{\int_{0}^{\infty} y(\xi)\left(\xi^{-2 \mu} H_{2 n}^{(-\mu)}(\xi)\right) \xi^{2 \mu} e^{-\xi^{2}} d \xi}{2^{4 n-1} n ! \Gamma(n-\mu+1)}
$$

We remark that the transformation $y=x^{-1-2 \mu} z$ leads to the same results. 


\section{LEFT-DEFINITE SPACES}

A recent topic of interest has been the setting of the differential operators such as $l$ and $m$ in a Sobolev space generated by the left side of the respective differential equations, hence left-definite spaces. Results in this kind of setting are now fairly well known (see [3, 4, 6, 7, 8, 9]), and so we merely state the results as they apply to the Hermite operators $l$ and $m$, rather than burden the reader with unnecessary details.

The even problem. We add a constant $k>0$ to the operator $l, l_{k}=l+k$, so that 0 is not in the spectrum of $l_{k}$, and so the Sobolev norm we are about to define will be a genuine norm. Thus for $\mu>-1 / 2$,

$$
l_{k} y=\frac{1}{2}\left(x^{2 \mu} e^{-x^{2}}\right)^{-1}\left(-\left(x^{2 \mu} e^{-x^{2}} y^{\prime}\right)^{\prime}+2 k x^{2 \mu} e^{-x^{2}} y\right) .
$$

$l_{k} y=(2 n+k) y$ when $y=H_{2 n}^{(\mu)}(x)$. Recall that there is no boundary condition required at $\infty$. The boundary condition at $x=0$ is

$$
\lim _{x \rightarrow 0} x^{2 \mu} e^{-x^{2}} y^{\prime}(x)=0
$$

Consider

$$
\int_{0}^{\infty}\left(l_{k} y\right) \bar{z} x^{2 \mu} e^{-x^{2}} d x=\frac{1}{2} \int_{0}^{\infty}\left[-\left(x^{2 \mu} e^{-x^{2}} y^{\prime}\right)^{\prime} \bar{z}+2 k x^{2 \mu} e^{-x^{2}} y \bar{z}\right] d x
$$

Integrating the first term on the right by parts gives

$$
-\left.\frac{1}{2}\left(x^{2 \mu} e^{-x^{2}} y^{\prime}\right) \bar{z}\right|_{0} ^{\infty}+\frac{1}{2} \int_{0}^{\infty}\left[x^{2 \mu} e^{-x^{2}} y^{\prime} \bar{z}^{\prime}+2 k x^{2 \mu} e^{-x^{2}} y \bar{z}\right] d x .
$$

We use the integral to define a new inner product and Hilbert (Sobolev) space. We show that the first, integrated terms vanish at 0 and $\infty$, provided $l_{k} y$ and $z$ lie in the new space and $y$ satisfies the boundary condition at 0 .

6.1. Definition. We denote by $H^{1}\left(0, \infty ; \frac{1}{2} x^{2 \mu} e^{-x^{2}}, k x^{2 \mu} e^{-x^{2}}\right)$ the complete inner product space generated by the inner product

$$
\langle y, z\rangle_{H^{1}}=\frac{1}{2} \int_{0}^{\infty}\left[x^{2 \mu} e^{-x^{2}} y^{\prime} \bar{z}^{\prime}+2 k x^{2 \mu} e^{-x^{2}} y \bar{z}\right] d x .
$$

We abbreviate the space name by $H_{e}^{1}$.

6.2. Definition. We denote by $\mathscr{D}_{k e}$ those elements $y$ in $H_{e}^{1}$ which satisfy the following.

(a) $y$ is absolutely continuous on all closed subintervals of $(0, \infty)$.

(b) $x^{2 \mu} e^{-x} y^{\prime}$ is absolutely continuous on all close subintervals of $(0, \infty)$.

(c) $l_{k} y$ exists a.e. and is in $H_{e}^{1}$.

(d) $\lim _{x \rightarrow 0} x^{2 \mu} e^{-x} y^{\prime}(x)=0$.

We define the operator $\mathscr{L}_{k}$ by setting $\mathscr{L}_{k} y=l_{k} y$ for all $y$ in $\mathscr{D}_{k e}$.

6.3. Theorem. For all $y$ in $\mathscr{D}_{k e}$ and $z$ in $H_{e}^{1}$,

$$
\begin{aligned}
& \lim _{x \rightarrow 0} x^{2 \mu} e^{-x^{2}} y^{\prime}(x) \bar{z}(x)=0, \\
& \lim _{x \rightarrow \infty} x^{2 \mu} e^{-x^{2}} y^{\prime}(x) \bar{z}(x)=0 .
\end{aligned}
$$


Proof. The limit at 0 follows from [9, Theorem 4.1]. The limit at $\infty$ follows from [6, Theorem 6.1].

Theorem 6.3 establishes

6.4. Theorem (Dirichlet formula). For all $y$ in $\mathscr{D}_{k e}$ and $z$ in $H_{e}^{1}$,

$$
\int_{0}^{\infty}\left(l_{k} y\right) \bar{z} x^{2 \mu} e^{-x^{2}} d x=\frac{1}{2} \int_{0}^{\infty}\left[x^{2 \mu} e^{-x^{2}} y^{\prime} \bar{z}^{\prime}+2 k x^{2 \mu} e^{-x^{2}} y \bar{z}\right] d x
$$

In inner product notation this is

$$
\langle(L+k) y, z\rangle_{L^{2}}=\langle y, z\rangle_{H_{e}^{1}} .
$$

Well-known machinery now establishes the following results, which we merely state. References at the beginning of this section contain the proofs.

\subsection{Theorem.}

(a) $\mathscr{D}_{k e}$ is dense in $H_{e}^{1}$.

(b) $\mathscr{L}_{k}$ is selfadjoint in $H_{e}^{1}$.

(c) $\mathscr{L}_{k}^{-1}$ exists and is bounded on $H_{e}^{1}$.

(d) The spectral resolutions associated with the identity, $\mathscr{L}_{k}^{-1}$ and $\mathscr{L}_{k}$ are the same as those associated with the identity, $(L+k)^{-1}$ and $(L+k)$ in Corollary 2.4.

A slight renorming of the Hermite polynomials $\left\{H_{2 n}^{(\mu)}(x)\right\}_{n=0}^{\infty}$ is needed, but the results are the same.

The odd problem. We add a positive constant $k>3$ to the operator $m$, $m_{k}=m+k$, so that 0 is not in the spectrum of $m_{k}$, and so that the Sobolev norm we are about to define will be a genuine. We must assume that $\mu>-1 / 2$, instead of the value $-3 / 2$ assumed earlier, so that the Hermite polynomials $\left\{H_{2 n+1}^{\infty}(x)\right\}_{n=0}^{\infty}$ lie in the Sobolev space. Thus for $\mu>-1 / 2$,

$$
m_{k} y=\frac{1}{2}\left(x^{2 \mu} e^{-x^{2}}\right)^{-1}\left(-\left(x^{2 \mu} e^{-x^{2}} y^{\prime}\right)^{\prime}+\left(2 \mu x^{2 \mu-1}+2 k x^{2 \mu}\right) e^{-x^{2}} y\right) .
$$

$m_{k} y=(2 n+1+k) y$ when $y=H_{2 n+1}^{(\mu)}(x)$. Recall that there is no boundary condition required at $\infty$. The boundary condition at $x=0$ is

$$
\lim _{x \rightarrow 0} x^{2 \mu} e^{-x^{2}}\left(x \frac{d y}{d x}-y\right)=0 \text {. }
$$

Consider

$$
\int_{0}^{\infty}\left(m_{k} y\right) \bar{z} x^{2 \mu} e^{-x^{2}} d x=\frac{1}{2} \int_{0}^{\infty}\left[-\left(x^{2 \mu} e^{-x^{2}} y^{\prime}\right)^{\prime} \bar{z}+\left(2 \mu x^{2 \mu-2}+2 k x^{2 \mu}\right) e^{-x^{2}} y \bar{z}\right] d x .
$$

Integrating the first term on the right by parts gives

$$
-\left.\frac{1}{2}\left(x^{2 \mu} e^{-x^{2}} y^{\prime}\right) \bar{z}\right|_{0} ^{\infty}+\frac{1}{2} \int_{0}^{\infty}\left[x^{2 \mu} e^{-x^{2}} y^{\prime} \bar{z}^{\prime}+\left(2 \mu x^{2 \mu-2}+2 k x^{2 \mu}\right) e^{-x^{2}} y \bar{z}\right] d x
$$

We use the integral to define a new inner product and Hilbert (Sobolev) space. We show that the first, integrated terms vanish at 0 and $\infty$, provided $m_{k} y$ and $z$ lie in the new space and $y$ satisfies the boundary condition at 0 . 
6.6. Definition. We denote by $H^{1}\left(0, \infty ; \frac{1}{2} x^{2 \mu} e^{-x^{2}},\left(\mu x^{2 \mu-2}+k x^{2 \mu}\right) e^{-x^{2}}\right)$ the complete inner product space generated by the inner product

$$
\langle y, z\rangle_{H^{1}}=\frac{1}{2} \int_{0}^{\infty}\left[x^{2 \mu} e^{-x^{2}} y^{\prime} \bar{z}^{\prime}+\left(2 \mu x^{2 \mu-2}+2 k x^{2 \mu}\right) e^{-x^{2}} y \bar{z}\right] d x .
$$

We abbreviate the space name by $H_{0}^{1}$.

6.7. Definition. We denote by $\mathscr{D}_{k 0}$ those elements $y$ in $H_{0}^{1}$ which satisfy the following.

(a) $y$ is absolutely continuous on all closed subintervals of $(0, \infty)$.

(b) $x^{2 \mu} e^{-x^{2}} y^{\prime}$ is absolutely continuous on all closed subintervals of $(0, \infty)$.

(c) $m_{k} y$ exists a.e. and is in $H_{0}^{1}$.

(d) $\lim _{x \rightarrow 0} x^{2 \mu} e^{-x^{2}}\left(x \frac{d \mu}{d x}-y\right)=0$

We define the operator $\mathscr{M}_{k}$ by setting $\mathscr{M}_{k} y=m_{k} y$ for all $y$ in $\mathscr{D}_{k 0}$.

6.8. Theorem. For all $y$ in $\mathscr{D}_{k 0}$ and $z$ in $H_{0}^{1}$,

$$
\begin{aligned}
& \lim _{x \rightarrow 0} x^{2 \mu} e^{-x^{2}} y^{\prime}(x) \bar{z}(x)=0, \\
& \lim x^{2 \mu} e^{-x^{2}} y^{\prime}(x) \bar{z}(x)=0 .
\end{aligned}
$$

Proof. Both limits follow from [6, Theorem 6.1].

Theorem 6.8 establishes

6.9. Theorem (Dirichlet formula). For all $y$ in $\mathscr{D}_{k 0}$ and $z$ in $H_{0}^{1}$,

$$
\int_{0}^{\infty}\left(m_{k} y\right) \bar{z} x^{2 \mu} e^{-x^{2}} d x=\frac{1}{2} \int_{0}^{\infty}\left[e^{-x^{2}} y^{\prime} \bar{z}^{\prime}+\left(2 \mu x^{2 \mu-2}+2 k x^{2 \mu}\right) e^{-x^{2}} y \bar{z}\right] d x .
$$

In inner product notation this is

$$
\langle(M+k) y, z\rangle_{L^{2}}=\langle y, z\rangle_{H_{0}^{1}} .
$$

Well-known machinery now establishes the following results, which we merely state. References at the beginning of this section contain the proofs.

\subsection{Theorem.}

(a) $\mathscr{D}_{k 0}$ is dense in $H_{0}^{1}$.

(b) $\mathscr{M}_{k}$ is selfadjoint in $H_{e}^{1}$.

(c) $\mathscr{M}_{k}^{-1}$ exists and is bounded on $H_{0}^{1}$.

(d) The spectral resolutions associated with the identity, $\mathscr{M}_{k}^{-1}$ and $\mathscr{M}_{k}$ are the same as those associated with the identity, $(M+k)^{-1}$ and $M+k$ in Corollary 3.4.

A slight renorming of the Hermite polynomials $\left\{H_{2 n+1}^{(\mu)}(x)\right\}_{n=0}^{\infty}$ is needed, but the results are the same.

\section{LEFT-DEFINITE SHIFTED SPACES}

It is in some ways not surprising that there is a left-definite theory for the Hermite operators $l$ and $m$ when $\mu<-1 / 2$. However, if the details are examined it becomes clear that the possibility has no guarantee. The problem, of course, is that under most transformations the left-definite norm is no longer a norm of the same kind. Luckily it is preserved in the Hermite cases. 
The even problem. We first note that, just as in the $L^{2}$ theory, when $\mu<1 / 2$, the operator

$$
l_{k} y=\frac{1}{2}\left(x^{2 \mu} e^{-x^{2}}\right)^{-1}\left(-\left(x^{2 \mu} e^{-x^{2}} y^{\prime}\right)^{\prime}+2 k x^{2 \mu} e^{-x^{2}} y\right)
$$

is transformed by $y=x^{-2 \mu} Y$ into

$$
\begin{aligned}
\widetilde{m}_{k+2 \nu} Y=\frac{1}{2}\left(x^{2 \nu} e^{-x^{2}}\right)^{-1}\left(-\left(x^{2 \nu} e^{-x^{2}} Y^{\prime}\right)^{\prime}\right. & \\
& \left.+\left(2 \nu x^{2 \nu-2}+[2 k+4 \nu] x^{2 \nu}\right) e^{-x^{2}} Y\right),
\end{aligned}
$$

where $\mu=-\nu$.

The left-definite inner product is likewise transformed. When $y=x^{-2 \mu} Y$, $z=x^{-2 \mu} Z$,

$$
\begin{aligned}
\frac{1}{2} \int_{0}^{\infty} & {\left[x^{2 \mu} e^{-x^{2}} y^{\prime} \bar{z}^{\prime}+2 k x^{2 \mu} e^{-x^{2}} y \bar{z}\right] d x } \\
= & \frac{1}{2} \int_{0}^{\infty}\left[x^{2 \nu} e^{-x^{2}} Y^{\prime} \bar{z}^{\prime}+\left(2 \nu x^{2 \nu-2}+(2 k+4 \nu) x^{2 \nu}\right) e^{-x^{2}} Y \bar{Z}\right] d x \\
& +\left.\nu\left(x^{2 \nu-1} e^{-x^{2}} Y \bar{Z}\right)\right|_{0} ^{\infty}
\end{aligned}
$$

We shall show that the last term vanishes.

7.1. Lemma. Let $Y$ be in $\mathscr{D}_{k 0}$ (Definition 6.7), and let $Z$ be in $H_{0}^{1}$ (Definition 6.6). Then

$$
\begin{aligned}
& \lim _{x \rightarrow 0} x^{2 \nu-1} e^{-x^{2}} Y Z=0, \\
& \lim _{x \rightarrow \infty} x^{2 \nu-1} e^{-x^{2}} Y Z=0 .
\end{aligned}
$$

Proof. First examine the limit at 0 , which must exist. Suppose it is not 0 . Then

$$
\lim _{x \rightarrow 0} x^{2 \nu} e^{-x^{2}}\left(x Y^{\prime}-Y\right)=0
$$

and

$$
\lim _{x \rightarrow 0} x^{2 \nu-1} e^{-x^{2}} Y Z=k \neq 0 .
$$

We know (temporarily letting $Y=\bar{Z}$ ) that

$$
\lim _{x \rightarrow 0} x^{2 \nu-1} e^{-x^{2}} Y^{2}=k_{1} \text {, }
$$

and

$$
\lim _{x \rightarrow 0} x^{2 \nu} e^{-x^{2}} Z^{2}=k_{2}
$$

Thus $k^{2}=k_{1} k_{2}$, and $k= \pm \sqrt{k_{1} k_{2}}$. If either $k_{1}$ or $k_{2}$ is 0 , we are done. So assume neither is 0 . Then for any $\epsilon>0$

$$
-\epsilon<\frac{x^{2 \nu} e^{-x^{2}}\left(x Y^{\prime}-Y\right)}{x^{2 \nu-1} e^{-x^{2}} Y^{2}}<\epsilon
$$

when $x$ is close to 0 . Thus

$$
-\epsilon / x<\left(Y-x Y^{\prime}\right) / Y^{2}<\epsilon / x
$$


Integrate from $x$ to $c$ (both near 0$)$.

$$
\epsilon(\ln x-\ln c)<c / Y(c)-x / Y(x)<\epsilon(\ln c-\ln x) .
$$

This implies

$$
\begin{aligned}
& {\left[\left(\frac{c}{Y(c)}+\epsilon \ln c\right) x^{-1}-(\epsilon \ln x) x^{-1}\right]^{-1}} \\
& \quad<Y(x)<\left[\left(\frac{c}{Y(c)}-\epsilon \ln c\right) x^{-1}+(\epsilon \ln x) x^{-1}\right]^{-1} .
\end{aligned}
$$

But the lower bound approaches 0 through positive values, while the upper bound approaches 0 through negative values. We have a contradiction.

The limit of $x^{2 \nu-1} e^{-x^{2}}|Y|^{2}$ as $x$ approaches $\infty$ also exists. If it is $k_{1} \neq 0$, then $x^{2 \nu} e^{-x^{2}}|Y|^{2} \simeq k_{1} / x$. Thus implies that

$$
\int^{\infty} x^{2 \nu} e^{-x^{2}}|Y|^{2} d x=\infty
$$

again a contradiction.

7.2. Definition. We denote by $\widetilde{D}_{k e}$ those elements $y$ in $H_{e}^{1}, \mu<\frac{1}{2}$, which satisfy the following.

(a) $y$ is absolutely continuous on all closed subintervals of $(0, \infty)$.

(b) $x^{2 \mu} e^{-x^{2}} y^{\prime}$ is absolutely continuous on all closed intervals of $(0, \infty)$.

(c) $l_{k} y$ exists a.e. and is in $H_{e}^{1}, \mu<1 / 2$.

(d) $\lim _{x \rightarrow 0} e^{-x^{2}}\left(x y^{\prime}(x)+[2 \mu+1] y(x)\right)=0$.

We define the operator $\widetilde{\mathscr{L}}_{k}$ by setting $\widetilde{\mathscr{L}}_{k} y=l_{k} y$ for all $y$ in $\widetilde{\mathscr{D}}_{k e}$.

We have established the following connection between norms:

7.2. Lemma. Let $y$ be in $\mathscr{D}_{k e}$ and $z$ be in $H_{e}^{1}$. Let $y=x^{-2 \mu} Y, z=x^{-2 \mu} Z$, $\mu<1 / 2$. Then, with $\mu=-\nu$,

$$
\begin{aligned}
& \frac{1}{2} \int_{0}^{\infty}\left[x^{2 \mu} e^{-x^{2}} y^{\prime} \bar{z}^{\prime}+2 k x^{2 \mu} y \bar{z}\right] d x \\
& \quad=\frac{1}{2} \int_{0}^{\infty}\left[x^{2 \nu} e^{-x^{2}} Y^{\prime} \bar{Z}^{\prime}+\left(2 \nu x^{2 \nu-2}+(2 k+4 \nu) x^{2 \nu}\right) e^{-x^{2}} Y \bar{Z}\right] d x .
\end{aligned}
$$

The second norm is equivalent to the norm in $H_{0}^{1}$ with $\mu=-\nu$.

Now the last integral equals

$$
\int_{0}^{\infty}\left(m_{k+2 \nu} Y\right) \bar{Z} x^{2 \nu} e^{-x^{2}} d x
$$

If the shift is undone, this equals

$$
\int_{0}^{\infty}\left(l_{k} y\right) \bar{z} x^{2 \mu} e^{-x^{2}} d x
$$

In the $L^{2}$ setting, this corresponds to

$$
\int_{0}^{\infty}(\tilde{L}+k) y \bar{z} x^{2 \mu} e^{-x^{2}} d x
$$

Assembling all this together, we have 
7.3. Theorem (Dirichlet formula). For all $y$ in $\widetilde{\mathscr{D}}_{k e}$ and let $z$ be in $H_{e}^{1}$, $\mu<\frac{1}{2}$, we have

$$
\int_{0}^{\infty}\left(l_{k} y\right) \bar{z} x^{2 \mu} e^{-x^{2}} d x=\frac{1}{2} \int_{0}^{\infty}\left[x^{2 \mu} e^{-x^{2}} y^{\prime} \bar{z}^{\prime}+2 k x^{2 \mu} e^{-x^{2}} y \bar{z}\right] d x .
$$

In inner product notation this is $\langle(\tilde{L}+k) y, z\rangle_{L^{2}}=\langle y, z\rangle_{H_{e}^{1}}$.

We again employ the machinery of previous works to derive the following results.

\subsection{Theorem.}

(a) $\widetilde{\mathscr{D}}_{k e}$ is dense in $H_{e}^{1}$.

(b) $\widetilde{\mathscr{L}}_{k}$ is selfadjoint in $H_{e}^{1}$.

(c) $\widetilde{\mathscr{L}}_{k}^{-1}$ exists and is bounded on $H_{e}^{1}$.

(d) The spectral resolutions associated with the identity, $\widetilde{\mathscr{L}}_{k}^{-1}$ and $\widetilde{\mathscr{L}}_{k}$ are the same as those associated with the identity $(\widetilde{\mathscr{L}}+k)^{-1}$ and $\widetilde{\mathscr{L}}+k$ in Corollary 5.4.

The odd problem. Here to we note that, just as in the $L^{2}$ theory, when $\mu<$ $1 / 2$, the operator

$$
m_{k} y=\frac{1}{2}\left(x^{2 \mu} e^{-x^{2}}\right)^{-1}\left(-\left(x^{2 \mu} e^{-x^{2}} y^{\prime}\right)^{\prime}+\left(2 \mu x^{2 \mu-2}+2 k x^{2 \mu}\right) e^{-x^{2}} y\right)
$$

is transformed by $y=x^{-2 \mu} Y$ into

$$
\tilde{l}_{k+2 \nu} Y=\frac{1}{2}\left(x^{2 \nu} e^{-x^{2}}\right)^{-1}\left(-\left(x^{2 \nu} e^{-x^{2}} Y^{\prime}\right)^{\prime}+(2 k+4 \nu) x^{2 \nu} e^{-x^{2}} Y\right)
$$

where $\mu=-\nu$.

The left-definite inner product is likewise transformed. When $y=x^{-2 \mu} Y$ and $z=x^{-2 \mu} Z$,

$$
\begin{aligned}
\frac{1}{2} \int_{0}^{\infty} & {\left[x^{2 \mu} e^{-x^{2}} y^{\prime} \bar{z}^{\prime}+\left(2 \mu x^{2 \mu-2}+2 k x^{2 \mu}\right) e^{-x^{2}} y \bar{z}\right] d x } \\
= & \frac{1}{2} \int_{0}^{\infty}\left[x^{2 \nu} e^{-x^{2}} Y^{\prime} \bar{Z}^{\prime}+\left(2 k+4 \nu x^{2 \nu}\right) e^{-x^{2}} Y Z\right] d x \\
& +\left.\nu\left(x^{2 \nu-1} e^{-x^{2}} Y Z\right)\right|_{0} ^{\infty} .
\end{aligned}
$$

We shall show that the last term vanishes.

7.5. Lemma. Let $Y$ be in $\mathscr{D}_{k e}$ (Definition 6.2) and let $Z$ be in $H_{e}^{1}$ (Definition 6.1). Then

$$
\begin{aligned}
& \lim _{x \rightarrow 0} x^{2 \nu-1} e^{-x^{2}} Y Z=0, \\
& \lim _{x \rightarrow \infty} x^{2 \nu-1} e^{-x^{2}} Y Z=0 .
\end{aligned}
$$

Proof. The verification of the second limit at $\infty$ is the same as in Lemma 7.1. The first involves only a slight variation on the previous argument. We establish that if

$$
\lim _{x \rightarrow 0} x^{2 \nu} Y^{\prime}(x)=0
$$


and

$$
\lim _{x \rightarrow 0} x^{2 \nu-1} Y^{2}(x)=k \neq 0
$$

then for any $\epsilon>0$

$$
-\epsilon / x<Y^{\prime} / Y^{2}<\epsilon / x,
$$

when $x$ is close to 0 . Integrate from $x$ to $c$ to find

$$
-\epsilon(\ln c-\ln x)<Y(x)^{-1}-Y(c)^{-1}<\epsilon(\ln c-\ln x)
$$

This implies

$$
[1 / Y(c)=\epsilon \ln c-\epsilon \ln x]^{-1}<Y(x)<[1 / Y(c)=\epsilon \ln c+\epsilon \ln x]^{-1} .
$$

but the lower bound approaches 0 through negative values, while the lower bound approaches 0 through positive values. We have a contradiction.

7.6. Definition. We denote by $\widetilde{\mathscr{D}}_{k 0}$ those elements $y$ in $H_{0}^{1}, \mu<\frac{1}{2}$, which satisfy the following.

(a) $y$ is absolutely continuous on all closed subintervals of $(0, \infty)$.

(b) $x^{2 \mu} e^{-x^{2}} y^{\prime}$ is absolutely continuous on all closed subintervals of $(0, \infty)$.

(c) $m_{k} y$ exists a.e. and is in $H_{0}^{1}, \mu<\frac{1}{2}$.

(d) $\lim _{x \rightarrow 0} e^{-x^{2}}\left(y^{\prime}(x)+2 \mu x^{-1} y(x)\right)=0$.

We define the operator $\widetilde{\mathscr{M}_{k}}$ by setting $\widetilde{\mathscr{M}}_{k} y=m_{k} y$ for all $y$ in $\widetilde{\mathscr{D}}_{k 0}$.

We have established the following connection between norms:

7.7. Lemma. Let $y$ be in $\widetilde{\mathscr{D}}_{k 0}$ and $z$ be in $H_{0}^{1}$. Let $y=x^{-2 \mu} Y, z=x^{-2 \mu} Z$, $\mu<\frac{1}{2}$. Then, with $\mu=-\nu$,

$$
\begin{aligned}
& \frac{1}{2} \int_{0}^{\infty}\left[x^{2 \mu} e^{-x^{2}} y^{\prime} \bar{z}^{\prime}+\left(2 \mu x^{2 \mu-2}+2 k x^{2 \mu}\right) e^{-x^{2}} y \bar{z}\right] d x \\
& \quad=\frac{1}{2} \int_{0}^{\infty}\left[x^{2 \nu} e^{-x^{2}} Y^{\prime} \bar{Z}^{\prime}+(2 k+4 \nu) x^{2 \nu} e^{-x^{2}} Y Z\right] d x
\end{aligned}
$$

The second is the equivalent to the norm in $H_{e}^{1}$ with $\mu=-\nu$.

Now the last integral equals

$$
\int_{0}^{\infty}\left(l_{k+2 \nu} Y\right) \bar{Z} x^{2 \nu} e^{-x^{2}} d x
$$

If the shift is undone, this equals

$$
\int_{0}^{\infty}\left(m_{k} y\right) \bar{z} e^{-x^{2}} d x
$$

In the $L^{2}$ setting this corresponds to

$$
\int_{0}^{\infty}(\widetilde{M}+k) y \bar{z} x^{2 \mu} e^{-x^{2}} d x .
$$

Assembling this together, we have 
7.8. Theorem (Dirichlet formula). For all $y$ in $\widetilde{\mathscr{D}}_{k 0}$ and $z$ in $H_{0}^{1}, \mu<\frac{1}{2}$, we have

$$
\int_{0}^{\infty}\left(m_{k} y\right) \bar{z} x^{2 \mu} e^{-x^{2}} d x=\frac{1}{2} \int_{0}^{\infty}\left[x^{2 \mu} e^{-x^{2}} y^{\prime} \bar{z}^{\prime}+\left(2 \mu x^{2 \mu-2}+2 k x^{2 \mu}\right) e^{-x^{2}} y \bar{z}\right] d x .
$$

In inner product notation this is $\langle(\widetilde{M}+k) y, z\rangle_{L^{2}}=\langle y, z\rangle_{H_{0}^{1}}$.

For the last time we employ the machinery of previous works to derive the following results.

\subsection{Theorem.}

(a) $\widetilde{\mathscr{D}}_{k 0}$ is dense in $H_{0}^{1}$.

(b) $\widetilde{\mathscr{M}_{k}}$ is selfadjoint in $H_{0}^{1}$.

(c) $\widetilde{\mathscr{M}}_{k}^{-1}$ exists and is bounded on $H_{0}^{1}$.

(d) The spectral resolutions associated with the identity, $\widetilde{\mathscr{M}}_{k}^{-1}$ and $\widetilde{\mathscr{M}_{k}}$ are the same as those associated with the identity, $(\widetilde{M}+k)^{-1}$ and $\widetilde{M}+k$ in Corollary 5.8.

\section{THE MACHINERY DEVELOPED PREVIOUSLY}

By now the author is sure the reader is rather frustrated with the lack of proofs for many of the results stated and the reference to previous results. To ease the frustration we give an outline of that theory.

We begin with the Dirichlet formula. We use $L$ to denote an $L^{2}$ operator, $\mathscr{L}$ to denote its restriction to the appropriate $H^{1}$ space. So for $y$ in $\mathscr{D}_{\mathscr{L}}$ and $z$ in $H^{1}$,

$$
\langle L y, z\rangle_{L^{2}}=\langle y, z\rangle_{H^{1}}
$$

Note that the element $z$ is rather arbitrary. Replacing $z$ by $\mathscr{L} z$, we have a second Dirichlet formula

$$
\langle L y, L z\rangle_{L^{2}}=\langle y, \mathscr{L} z\rangle_{H^{1}} .
$$

Since this also equals $\langle\mathscr{L} y, z\rangle_{H^{1}}$, we find

$$
\langle\mathscr{L} y, z\rangle_{H^{1}}=\langle y, \mathscr{L} z\rangle_{H^{1}} .
$$

$\mathscr{L}$ is symmetric.

Letting $z=y, \mathscr{L} y=f, y=\mathscr{G} f$, where $\mathscr{G}$ represents the inverse of $L$ in $L^{2}$, the Dirichlet formula gives

$$
\langle f, \mathscr{G} f\rangle_{L^{2}}=\langle\mathscr{G} f, \mathscr{G} f\rangle_{H^{1}}
$$

When $\|f\|_{L^{2}} \leq k\|f\|_{H^{1}}, k>0$, thus implies

$$
k\|f\|_{H^{1}}^{2}\|\mathscr{G}\|_{L^{2}} \geqq\|\mathscr{G} f\|_{H^{1}}^{2} .
$$

If we divide by $\|f\|_{H^{1}}^{2}$ and take the supremium over $\|f\|_{H^{1}}$, we have

$$
\|\mathscr{G}\|_{H^{1}} \leq\left(k\|\mathscr{G}\|_{L^{2}}\right)^{1 / 2},
$$

and so $\mathscr{G}$ is bounded on $H^{1}$. Thus $\mathscr{L}$ is a maximally extended symmetric operator on $H^{1}$, and is therefore selfadjoint. 
We now have two spectral resolutions associated with $L$ and $\mathscr{L}$. Let us denote them by $S f$ and $\mathscr{S} f$. Both are in $L^{2}$.

The Dirichlet formula establishes for $f$ in $H^{1}$ and $y$ in $\mathscr{D}_{\mathscr{L}}$,

$$
\langle L y, S f\rangle_{L^{2}}=\langle L y, f\rangle_{L^{2}}=\langle y, f\rangle_{H^{1}}=\langle y, \mathscr{S} f\rangle_{H^{1}}=\langle L y, \mathscr{S} f\rangle_{L^{2}}
$$

Hence $\langle L y, S f-\mathscr{S} f\rangle_{L^{2}}=0$. Since $\{\overline{L y}\}$ spans all of $L^{2}, S f=\mathscr{S} f$ a.e. and thus in $L^{2}$ and $H^{1}$. There is only one spectral resolution.

We hope this outline is satisfactory.

\section{REFERENCES}

1. T. S. Chihara, An introduction to orthogonal polynomials, Gordon and Breach, New York, 1978.

2. E. A. Coddington and N. Levinson, Theory of ordinary differential equations, McGraw-Hill, New York, 1955.

3. M. Hazmirzaahmad, Jacobi polynomial expansions, J. Math. Anal. Appl. (to appear).

4. __ Laguerre polynomial expansions, J. Comput. Appl. Math. (to appear).

5. A. M. Krall, On the generalized Hermite polynomials $\left\{H_{n}^{(\mu)}\right\}_{n=0}^{\infty}, \mu<-1 / 2$, Indiana J. Math. 30 (1981), 73-78.

6. —,$H^{1}$ convergence of Fourier transforms, submitted.

7. — $H^{1}$ Bessel expansions, submitted.

8. __, Regular left-definite boundary value problems of even order, Quaestiones Math. 15 (1992), 105-118.

9. A. M. Krall and D. Race, Self-adjointness for the Weyl problem under an energy norm, submitted.

10. L. L. Littlejohn and A. M. Krall, Orthogonal polynomials and singular Sturm-Liouville systems. I, Rocky Mountain J. Math. 10 (1986), 435-479.

11. E. D. Rainville, Special functions, Macmillan, New York, 1960.

331 McAllister Building, The Pennsylvania State University, University Park, PennSYLVANIA 16802

E-mail address: krall@math.psu.edu 